\title{
Templated Growth of Metastable Polymorphs on Amorphous Substrates with Seed Layers
}

\author{
Yanbing Han, ${ }^{1,2,3}$ Ryan Trottier, ${ }^{4}$ Sebastian Siol, ${ }^{1, \$}$ Bethany Matthews, ${ }^{1,5}$ Matthew Young, ${ }^{1}$ \\ Charles B. Musgrave, ${ }^{4}$ Stephan Lany, ${ }^{1}$ Janet Tate, ${ }^{5}$ Qun Zhang,,${ }^{2, *}$ Aaron M. Holder, ${ }^{1,4}$ \\ and Andriy Zakutayev $\circledast^{1, \dagger}$ \\ ${ }^{1}$ Materials Science Center, National Renewable Energy Laboratory, Golden, Colorado 80401, USA \\ ${ }^{2}$ Department of Materials Science, Fudan University, Shanghai 200433, China \\ ${ }^{3}$ School of Physics and Microelectronics, ZhengZhou University, Zhengzhou 450052, China \\ ${ }^{4}$ Department of Chemical and Biological Engineering, University of Colorado, Boulder, Colorado 80309, USA \\ ${ }^{5}$ Department of Physics, Oregon State University, Corvallis, Oregon 97330, USA
}

(Received 12 June 2019; revised manuscript received 19 September 2019; published 8 January 2020)

\begin{abstract}
Metastable inorganic materials with unique properties are important in many practical applications, but their synthesis is often challenging. In physics, epitaxial stabilization, also known as pseudomorphic growth, is used to synthesize metastable polymorphs, but usually only as very thin films and on expensive single-crystal substrates. In chemistry, the templated growth of inorganic solid-state materials on selfassembled monolayers of organic molecules is reported. Bridging these two fields, here, we show that the synthesis of metastable polymorphs is possible up to large film thickness on amorphous substrates covered with thin inorganic seed layers that serve as templates. The stabilization of a 500-nm-thick metastable wurtzite (WZ) MnTe film by a 5-nm-thick ZnTe seed layer sputtered on amorphous glass substrates is experimentally demonstrated. Theoretical calculations explain this experimental observation by the small WZ polymorph energy relative to that of the ground-state nickeline (NC) structure of MnTe and a large lattice constant difference of the two. The resulting metastable WZ-MnTe polymorph exhibits a wide band gap of $2.7 \mathrm{eV}$ and a low hole density of $10^{12} \mathrm{~cm}^{-3}$, which is relevant to optoelectronic applications. These properties are in sharp contrast to those of the narrow-band-gap highly doped NC-MnTe with $1.3 \mathrm{eV}$ band gap and $10^{19} \mathrm{~cm}^{-3}$ hole density. The difference in hole density is due to the calculated difference in the formation energy of manganese vacancy acceptor defects. Overall, these results suggest that templated growth on amorphous substrates with seed layers can be used to synthesize metastable polymorphs of other materials, without the need for expensive single-crystal substrates.
\end{abstract}

DOI: 10.1103/PhysRevApplied.13.014012

\section{INTRODUCTION}

Metastable materials are ubiquitous in our lives, as they are used across a broad range of practical applications. Examples among inorganic solids include the diamond polymorph of carbon, austenitic steel alloys, $\mathrm{GaInP}_{2} \mathrm{com}-$ pounds in multijunction photovoltaics, and amorphous glass in building windows. One type of metastable materials of particular relevance to this paper are polymorphs; these are materials with the same composition, but different crystal structures from those of their stable counterparts. Metastable polymorphs are important for technological applications because they can have completely different

\footnotetext{
*zhangqun@fudan.edu.cn

†andriy.zakutayev@nrel.gov

¥Present address: Empa - Swiss Federal Laboratories for Materials Science and Technology, 8600 Dübendorf, Switzerland.
}

properties than those of their thermodynamically stable ground states. For example, it is theoretically predicted that the archetypical wide-band-gap (WBG) $n$-type semiconductor $\mathrm{ZnO}$ can be doped to form a $p$-type semiconductor using $\mathrm{Li}$, if its rock salt polymorph, rather than wurtzite ground state, is stabilized [1]. This is important because WBG $p$-type semiconductors are desirable for (opto-)electronic devices, such as solar cells, thinfilm transistors, photodetectors, and light-emitting diodes [2-4], but their $p$-type doping is often difficult to achieve [5-7]. Metastable polymorphs are also interesting from the point of view of synthesis science. Understanding how to synthesize polymorphs in a controlled way can dramatically increase the number of possible materials that can be used for the aforementioned technologies and many other practical applications.

One common approach to synthesize metastable polymorphs in thin-film form is epitaxial growth [8], also known as "pseudomorphic stabilization" [9]. This 
approach is based on the idea that the crystal structure of the film grown on the substrate is determined by the total free energy of the film-substrate system, which includes bulk and interface components. Such epitaxial growth has been used in the past for many materials, for example, to engineer functionality into complex oxides grown using molecular beam epitaxy (MBE) [10] by tuning of ferroelectric properties by strain energy [11] between singlecrystal substrates and thin films. As another example, films of high-energy rock salt polymorphs of GaN [12] and AlN [13] of up to 1-2 nm thick have been synthesized by epitaxial growth on TiN and $\mathrm{MgO}$. However, the broad use of this physical method for the synthesis of metastable polymorphs in thin-film form thus far has been limited by the use of expensive single-crystal substrates, the complexity of MBE methods, and the very small critical thickness of the films. A related chemical method for the synthesis of metastable materials is templated growth from solution on self-assembled monolayers, with the resulting structure (e.g., calcite, vaterite, and aragonite polymorphs of $\mathrm{CaCO}_{3}$ ) controlled by the selection of organic molecules $[14,15]$.

Recently, we reported that a metastable wurtzite polymorph of MnTe (WZ-MnTe) could be stabilized on amorphous indium zinc oxide ( $a$-IZO) coated glass [16]. Surprisingly, we found that the growth of WZ-MnTe on $a$ IZO was not limited to critical thickness and not influenced by the work function of the substrate. We hypothesized that WZ-MnTe stabilization might be due to interfusion at the WZ-MnTe/a-IZO interface. However, it remains unclear whether stabilization is due to chemical alloying and/or structural templating mechanisms. To investigate the chemical alloying mechanism, we synthesized $\mathrm{Mn}_{1-x} \mathrm{Zn}_{x} \mathrm{Te}$ on glass substrates and found that the WZ polymorph can be stabilized at $x>0.08-0.18$, depending on the growth temperature $\left(300-400^{\circ} \mathrm{C}\right)$ [17]. These experimental observations were theoretically explained by much larger mixing enthalpy of $\mathrm{Mn}_{1-x} \mathrm{Zn}_{x}$ Te alloys in the NC compared with the WZ structure, similar to the stabilization of "negative-pressure" WZ polymorphs in $\mathrm{MnSe}_{1-x} \mathrm{Te}_{x}$ heterostructural alloys [18]. However, the properties of the metastable WZ-MnTe polymorph synthesized in these $\mathrm{Mn}_{1-x} \mathrm{Zn}_{x} \mathrm{Te}$ and $\mathrm{MnSe}_{1-x} \mathrm{Te}_{x}$ experiments were modified by inclusion of $\mathrm{Zn}$ or Se alloying constituents, so alternative synthesis routes to WZ-MnTe were desirable. In addition, a contribution of the structural templating mechanism to WZ-MnTe stabilization remained unknown.

Here, we show that metastable WZ-MnTe can be synthesized using sputtering on amorphous substrates covered with ultrathin seed layers. Specifically, about 500-nmthick WZ-MnTe is sputtered on a 5-nm-thick ZnTe seed layer deposited on an amorphous fused silica substrate. Stabilization of the WZ phase is explained by the theoretical prediction of the polymorph energy difference as a function of the lattice constant. The resulting WZ-MnTe metastable polymorph shows a wide $2.1-2.7 \mathrm{eV}$ band gap and low $10^{12} \mathrm{~cm}^{-3}$ hole density; these values are considerably different from those of the $1.3 \mathrm{eV}$ band gap and $10^{19} \mathrm{~cm}^{-3}$ hole density of the thermodynamic groundstate nickeline (NC) structure. The drastic difference in electrical properties is explained by the $>1 \mathrm{eV}$ larger formation energy of acceptor-type manganese vacancies, as determined using first-principles defect calculations. Overall, these results demonstrate how templated growth on amorphous substrates with seed layers can be used to synthesize metastable polymorphs and expand the range of known wide-band-gap $p$-type materials for optoelectronic applications.

\section{METHODS}

Thin films reported in this work are deposited by a customized AJA combinatorial radiofrequency sputtering system from 50.8 -mm-diameter ZnTe $(99.99 \%$ purity) and MnTe (99.9\%) targets tilted relative to the substrate. The stationary $50.8 \times 50.8 \times 1.6 \mathrm{~mm}^{3}$ amorphous fused silica substrates are used to avoid the influence of substrate crystal structure on thin-film growth. The working pressure is 1.3 mTorr with an ultrahigh purity argon flow rate of $16 \mathrm{sccm}$. The chamber is pumped down to $1 \times 10^{-6}$ Torr before deposition. The deposition rate of $\mathrm{ZnTe}$ decreases from 5-10 to 3-6 $\mathrm{nm} / \mathrm{min}$ with increasing substrate temperature $\left(T_{\text {sub }}\right)$, while that of MnTe is in the range of 2-5 $\mathrm{nm} / \mathrm{min}$, regardless of temperature, as summarized in Table S1 within the Supplemental Material [30]. These rates, combined with 90-180 min deposition times, result in typical thicknesses of 450-500 nm WZ-MnTe films, and 300 min deposition times lead to 1260 -nm-thick films. More deposition details were described in our previous work [17].

To characterize the samples, $\mathrm{x}$-ray fluorescence (XRF, Fischerscope XDV-SDD) is utilized to determine the composition and thickness with a 3-mm-diameter spot size, and thus, confirms the MnTe films to be stoichiometric within the accuracy of the methods (0.1-1.0 at. \%). X-ray diffraction is measured using $\mathrm{Cu} K \alpha$ radiation on a Bruker D8 x-ray diffractometer, with a 2D detector in approximate two-theta geometry. Transmittance and reflectance are measured using a custom Ocean optics ultraviolet-tovisible (UV-vis) spectrometer system with wavelengths from 300 to $1000 \mathrm{~nm}$ and used to determine optical absorption. Carrier concentration and Hall mobility as a function of temperature are determined from the magnitude and sign of the Hall voltage and from electrical resistivity, all measured using a Lakeshore instrument (model 8425), under a magnetic field of $2 \mathrm{~T}$. We note that low hole density and large surface depletion width for WZ-MnTe, as well as possible magnetic character of the MnTe materials, can convolute analysis of the Hall effect measurement results. 
Secondary ion mass spectrometry (SIMS) is performed on a Cameca IMS-7f instrument using an oxygen primaryion beam at $10 \mathrm{kV}$ and a sample potential of $5 \mathrm{kV}$ with positive secondary ions. The cross-section TEM lamella of the $300^{\circ} \mathrm{C} \mathrm{MnTe} / \mathrm{ZnTe} / \mathrm{SiO}_{2}$ sample is prepared in a FEI Nova dual-beam instrument and analyzed under conventional TEM conditions to assess the lattice spacings. The results are analyzed using a custom COMBIgor analysis package for Igor Pro [19], and the raw data is available in the High Throughput Experimental Materials Database [20]. Detailed characterization information can also be found in our previous work [21].

The polymorph energies are calculated from first principles in the random-phase approximation (RPA), with a variational optimization of the wave function that correctly recovers the Mn- $d /$ Te- $p$ hybridization of the MnTe polymorphs [18]. Defect calculations are performed using the projector-augmented-wave method implemented within the VASP code [22-24], using the meta-GGA SCAN functional [25]. The results of our defect calculations using $\mathrm{PBE}+\mathrm{U}[26,27]$, with $U=3 \mathrm{eV}$ applied to $\mathrm{Mn}-d$ [28] are reported within the Supplemental Material [30] for reference. An energy cutoff of $800 \mathrm{eV}$ and minimum $k$-point grid densities of about 1000 per reciprocal atom are employed in all calculations to achieve total energy convergence to $0.005 \mathrm{eV} /$ atom. The defect calculations are performed on nickeline and wurtzite 72-atom supercells. To account for supercell finite-size effects in our calculations, we apply a correction scheme [29]. A linear scissor correction is applied to the results of the defect calculations to align the valence-band maximum and conduction-band minimum to the predicted $G W$ band gaps from the NREL MatDB computational materials database.

\section{RESULTS AND DISCUSSION}

\section{A. Composition and structure}

The XRD patterns of the chemically stoichiometric $\mathrm{MnTe} / \mathrm{ZnTe} / \mathrm{SiO}_{2}$ samples (MnTe on $5 \mathrm{~nm} \mathrm{ZnTe}$ on $a-\mathrm{SiO}_{2}$ substrate) and $\mathrm{MnTe} / \mathrm{SiO}_{2}$ control samples (MnTe on $a$-SiO $\mathrm{Sib}_{2}$ substrate) deposited at 300 and $400{ }^{\circ} \mathrm{C}$, all with thicknesses of 440-500 nm (Table S1 within the Supplemental Material [30]), are shown in Fig. 1(a). At $400^{\circ} \mathrm{C}$, only NC-MnTe is observed, regardless of the substrate, as expected, because this is the lowest energy polymorph of MnTe. However, at $300^{\circ} \mathrm{C}$, the XRD peaks of the $\mathrm{MnTe} / \mathrm{ZnTe} / a-\mathrm{SiO}_{2}$ samples can all be matched to the calculated WZ structure XRD reference pattern [Fig. 1(a)], whereas $\mathrm{MnTe} / a-\mathrm{SiO}_{2}$ remains in the NC structure [Fig. 1(a)]. All films are polycrystalline, with no single preferential orientation, which suggests that the substrate strain is fully relaxed. XRD results for other deposition and annealing temperatures, as well as $\mathrm{ZnTe}$ on $a-\mathrm{SiO}_{2}$, are shown in Figure S1 within the Supplemental Material [30]. For the $<300{ }^{\circ} \mathrm{C} \mathrm{MnTe}$ depositions, some $\mathrm{MnTe}_{2}$ phase
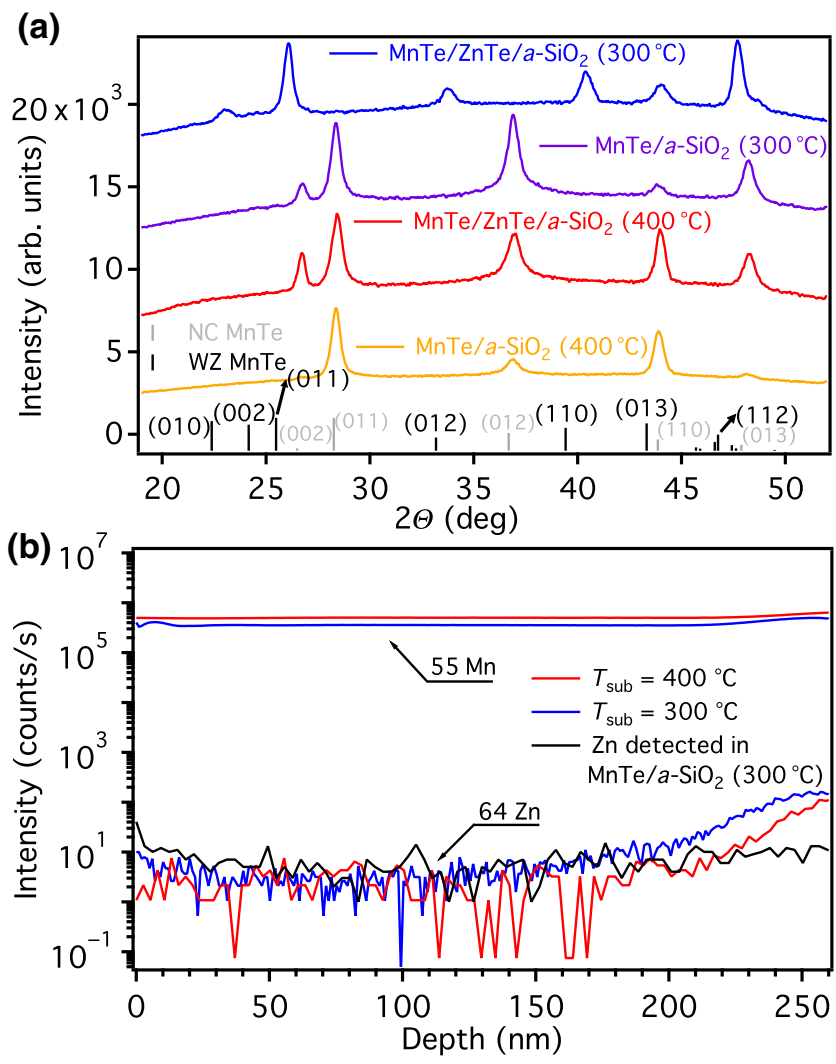

FIG. 1. (a) XRD patterns of 450-500-nm-thick MnTe thin films on $a-\mathrm{SiO}_{2}$ substrates as a function of temperature $\left(300 \mathrm{vs} 400^{\circ} \mathrm{C}\right)$ and seed layer (ZnTe vs none). (b) SIMS depth profiles of 250-nm-thick $\mathrm{MnTe} / \mathrm{ZnTe} / \mathrm{SiO}_{2}$ samples deposited at different temperatures $\left(300\right.$ vs $\left.400{ }^{\circ} \mathrm{C}\right)$. These results suggest stabilization of the WZ structure at lower temperatures by templated growth.

impurity is formed, whereas at $>300^{\circ} \mathrm{C} \mathrm{MnTe}$ forms in the $\mathrm{NC}$ structure. ZnTe remains in its ground-state zinc blende (ZB) structure, with preferential (111) orientation in the $200-300{ }^{\circ} \mathrm{C}$ range, and above $500{ }^{\circ} \mathrm{C}$ it does not adhere to the substrate. The $\mathrm{MnTe} / \mathrm{ZnTe} / \mathrm{SiO}_{2}$ sample deposited at $300^{\circ} \mathrm{C}$ and annealed at $400^{\circ} \mathrm{C}$ retains the $\mathrm{WZ}$ phase with a negligible amount of $\mathrm{NC}$ phase (Figure $\mathrm{S} 1$ within the Supplemental Material [30]).

Figure 1(b) summarizes the results of SIMS based on $250 \mathrm{~nm} \mathrm{MnTe} / \mathrm{ZnTe} / \mathrm{SiO}_{2}$ samples, showing the $\mathrm{Mn}$ and $\mathrm{Zn}$ signal intensity as a function of the distance from the sample surface. For samples deposited at both 300 and $400{ }^{\circ} \mathrm{C}$, a small $\mathrm{Zn}$ signal close to the $a-\mathrm{SiO}_{2}$ substrate $(10-100$ counts/s) is detected, but, for the majority of the film, no $\mathrm{Zn}$ signal is observed above the noise level $(1-10$ counts/s at depth $<200 \mathrm{~nm})$, which is the same as that for the MnTe samples. Complementary Auger electron spectroscopy (AES) depth profile measurements $(<0.1-1.0 \%$ detection limit $)$ do not reveal any oxygen or other major unintentional impurities in the bulk, as shown in Figure S2 within the Supplemental Material [30]. 
At both temperatures, it appears that the $\mathrm{Zn}$ depth profile in MnTe remains approximately the same, yet MnTe exhibits a totally different crystal structure: $\mathrm{WZ}$ at $300^{\circ} \mathrm{C}$ and $\mathrm{NC}$ at $400^{\circ} \mathrm{C}$ [Fig. 1(a)]. A cross-section microscopy image of the film/seed/substrate interface for the $300^{\circ} \mathrm{C}$ $\mathrm{MnTe} / \mathrm{ZnTe} / \mathrm{SiO}_{2}$ sample is shown in Figure $\mathrm{S} 2$ within the Supplemental Material [30] and confirms the WZ phase based on the lattice constant. The presence of the seed layer in Fig. 1(b) indicates that alloying through the thickness of the film is not responsible for the WZ-phase stabilization observed here. In addition, the overall $\mathrm{Zn}$ content in both samples determined from XRF is $<0.04$ cat. at. \%, which would lead to a mixture of $\mathrm{NC}$ and $\mathrm{WZ}$ phases, according to our previous reports [17]. Thus, there must be a different mechanism responsible for stabilization of the WZ-MnTe polymorph.

\section{B. Stabilization mechanism}

To understand the stabilization of WZ-MnTe on the ZnTe seed layer, we calculate the bulk equation of state (energy vs volume) for the two observed MnTe polymorphs WZ and NC [Fig. 2(a)] from first principles in RPA [18]. As shown in Fig. 2(b), the WZ polymorph of MnTe would be stable if its energy $\left(E_{\mathrm{pm}}^{\mathrm{WZ}}\right)$ were lower than that of the strain energy of the $\mathrm{NC}$ ground-state structure $\left(E_{\mathrm{st}}^{\mathrm{NC}}\right)$ :

$$
E_{\mathrm{pm}}^{\mathrm{WZ}}<E_{\mathrm{st}}^{\mathrm{NC}}+\Delta E_{\mathrm{if}}+\Delta E_{\mathrm{sf}}
$$

where $\Delta E_{\mathrm{if}}=E_{\mathrm{if}}^{\mathrm{NC}}-E_{\mathrm{if}}^{\mathrm{WZ}}$ and $\Delta E_{\mathrm{sf}}=E_{\mathrm{sf}}^{\mathrm{NC}}-E_{\mathrm{sf}}^{\mathrm{WZ}}$ are differences in the interface and surface energies, respectively, of the two polymorphs, which are typically smaller than those of the bulk energies $\left(<100 \mathrm{meV} / \mathrm{A}^{2}\right)$ [31]. The results of our calculations shown in Fig. 2(a) indicate that the strain energy of the NC polymorph at the WZ equilibrium volume $(1500 \mathrm{meV} / \mathrm{at})$ is much larger than this polymorph energy difference $(7 \mathrm{meV} / \mathrm{at})$ at the respective equilibrium volumes $\left(V_{\text {eq }}\right)$. Thus, we conclude that, when MnTe is deposited on the ZnTe seed layer, it minimizes the overall energy by adopting the WZ crystal structure, which is close in energy to the ground-state $\mathrm{NC}$ crystal structure. A detailed derivation of Eq. (1) and a schematic corresponding to the general case (Figure S3) are presented within the Supplemental Material [30].

Polymorph stabilization has been previously observed for metastable ZB-MgS films deposited on GaAs crystals [32], and for various metastable oxides and nitrides $[8,12]$ grown on single-crystal substrates, up to a small critical thickness, using complex MBE methods. The important differences between this study and all of these prior thinfilm reports are (a) thick $500 \mathrm{~nm}$ metastable WZ-MnTe layers, (b) layers are deposited using a simple sputtering technique, (c) 5-nm-thick ZnTe seed layers, and (d) inexpensive amorphous glass substrates are used. Such templated growth opens up a huge new space for the

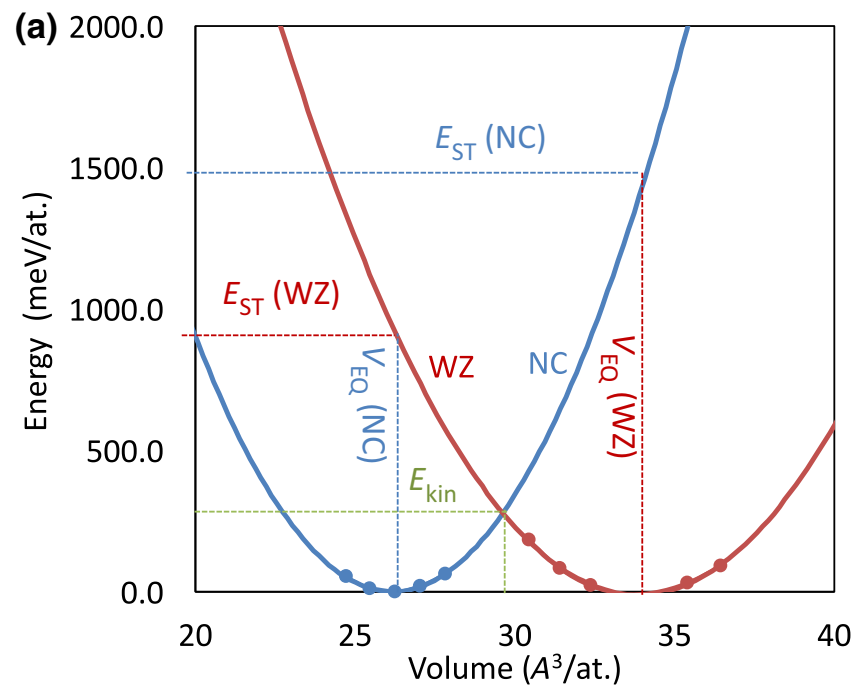

(b)

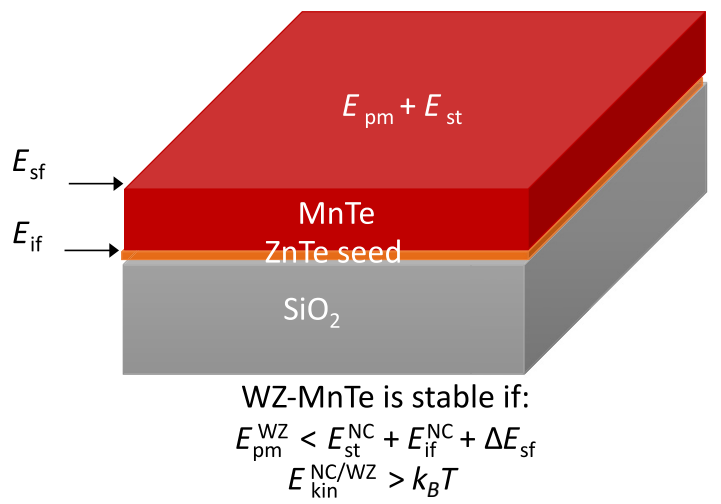

FIG. 2. (a) Calculated bulk equation of state for the WZ and $\mathrm{NC}$ polymorphs of MnTe. The polymorph energies are similar, but the strain energies are different. (b) Schematic illustration of the total energy of a MnTe thin film grown on a thin ZnTe seed deposited on an $a-\mathrm{SiO}_{2}$ substrate. WZ-MnTe can be stabilized if the WZ polymorph energy is smaller than that of the NC strain energy.

stabilization of high-energy polymorphs in materials systems, where lattice-matched single-crystal substrates are not available, complex deposition methods are not feasible, and thick films are required for practical applications. This discovery also highlights the importance of emerging tools for high-throughput studies of metastable materials, such as combinatorial substrate epitaxy [33].

\section{Optical and electrical properties}

One of the distinguishing features of the WZ polymorph of MnTe is its wide band gap, relative to that of the NC polymorph of MnTe. The transmittance spectra, corrected by the reflectance spectrum to avoid interference fringes, of WZ- and NC-MnTe deposited at $300^{\circ} \mathrm{C}$ are shown in Fig. 3(a). WZ-MnTe has a corrected transmittance of $90 \%$ in the wavelength range of $600-1000 \mathrm{~nm}$, in contrast to the nearly opaque NC-MnTe ground-state structure. The 

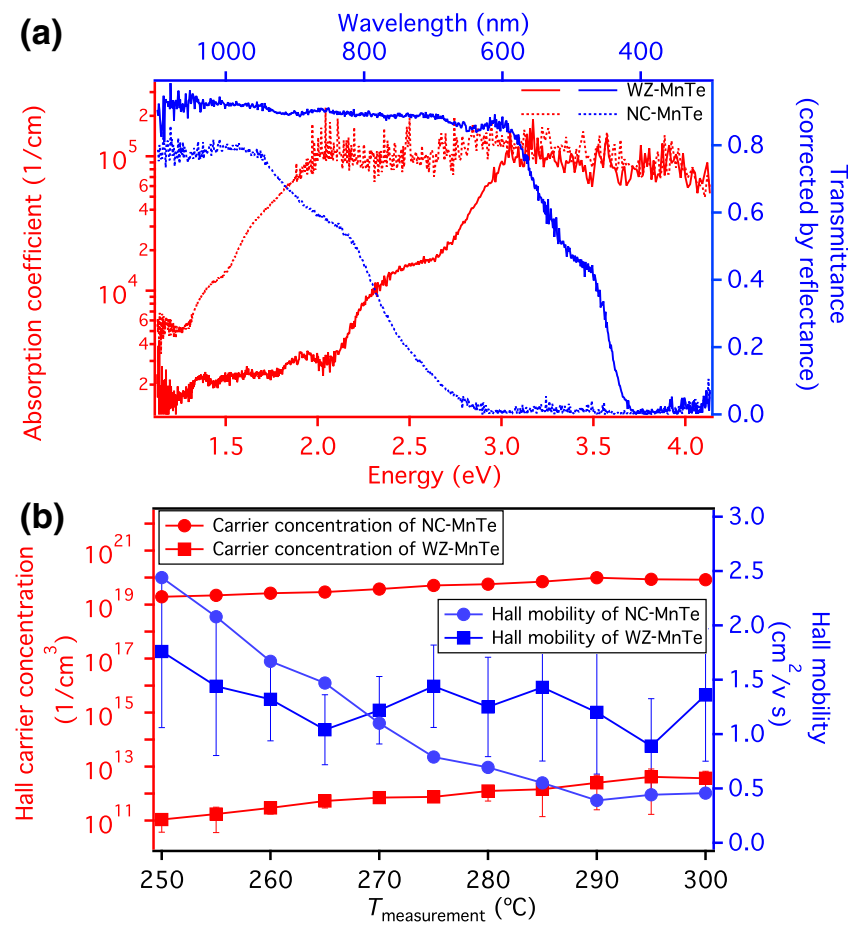

FIG. 3. (a) Transmittance versus wavelength and absorption versus energy for $\mathrm{WZ}$ and $\mathrm{NC}$ polymorphs of MnTe. The WZ phase has a wider band gap than that of the NC phase. (b) Hall concentration and mobility of WZ-MnTe and NC-MnTe as a function of measurement temperature. The WZ phase has a much lower carrier concentration compared with that of the NC phase.

corresponding absorption thresholds determined from the logarithmic plots of the absorption coefficient are approximately $2.7 \mathrm{eV}$ for WZ-MnTe (with some subthreshold absorption) and $1.3 \mathrm{eV}$ for NC-MnTe. This difference is consistent with the theoretically calculated values of band gaps reported in the NREL MatDB computational materials database. Absorption coefficient plots of MnTe samples grown on amorphous fused silica with or without a $\mathrm{ZnTe}$ seed layer are provided in Figure S4 within the Supplemental Material [30]. Not surprisingly, phase-pure WZ-MnTe exhibits a wide band gap $(2.7 \mathrm{eV})$, while the others have lower optical absorption onsets of $<1.3 \mathrm{eV}$ due to the $\mathrm{NC}-\mathrm{MnTe}$ and $\mathrm{MnTe}_{2}$ impurities.

The difference in the band gaps of WZ- and NC-MnTe also correlates with the dramatic change in the electrical properties of these semiconductors. Figure 3(b) summarizes the preliminary carrier concentration and mobility measurements by the Hall effect as a function of temperatures for the NC-MnTe and WZ-MnTe samples. Both samples show positive signs of the Hall coefficient, which indicates that the materials are $p$-type semiconductors. WZ-MnTe has a much lower hole concentration (approximately $10^{12} \mathrm{~cm}^{-3}$ ) than that of NC-MnTe (approximately $10^{19} \mathrm{~cm}^{-3}$ ). It is unlikely that extrinsic impurities, such as
$\mathrm{O}$ and $\mathrm{C}$, lead to such huge differences in hole concentration, since they are not observed by AES (Figure S2 within the Supplemental Material [30]) and the synthesis conditions for both polymorphs are similar. The low hole concentration, along with the wide band gap, is beneficial for application as a channel layer in thin-film transistors. The WZ-MnTe hole mobility value for our sputtered smallgrain polycrystalline samples is about $1.4 \mathrm{~cm}^{2} / \mathrm{Vs}$, which is comparable to that of NC-MnTe and to other known wide-band-gap $p$-type semiconductors used in thin-film transistors. We also note that the hole mobility is likely to be considerably higher for WZ-MnTe films with larger grains because the calculated hole effective mass for this material is quite low [17].

\section{Defect calculations}

The measured dramatic difference in the hole concentrations of $\mathrm{NC}$ and $\mathrm{WZ}$ polymorphs of MnTe can be explained by the difference in the native point-defect concentrations. Theoretical calculations shown in Fig. 4 suggest that WZMnTe has a higher formation enthalpy by about $1 \mathrm{eV}$ for acceptor-type manganese vacancy $\left(V_{\mathrm{Mn}}\right)$ defects relative to that of NC-MnTe, which is the main difference in defects

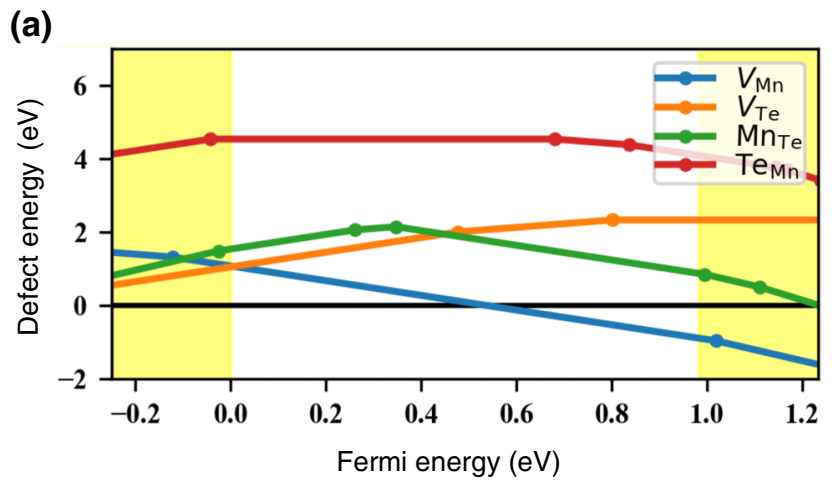

(b)

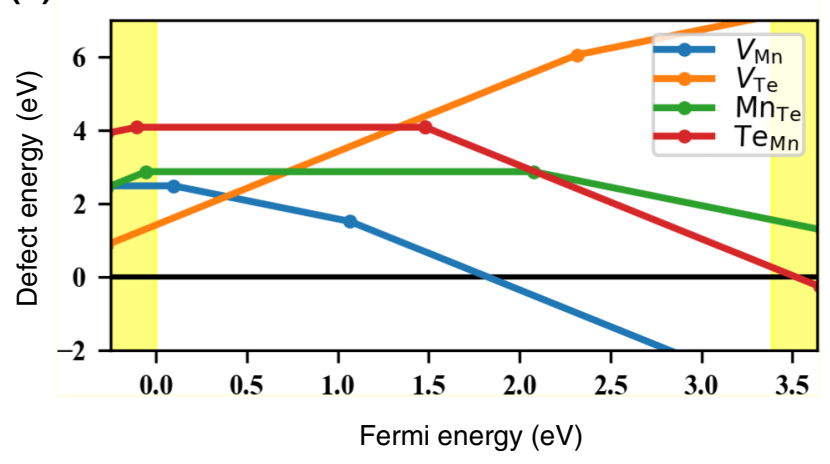

FIG. 4. Defect formation energies under Mn-rich conditions, as a function of Fermi level for MnTe in (a) nickeline and (b) wurtzite crystal structures. WZ-MnTe is expected to have a lower density of free holes based on the higher energy of the manganese vacancy acceptor defects compared with those of NC-MnTe. 
physics of the $\mathrm{WZ}$ and $\mathrm{NC}$ polymorphs. For both polymorphs, the $\mathrm{Mn}_{\mathrm{Te}}$ ambipolar antisite defects and the $V_{\mathrm{Te}}$ donors have similar energies to those of $V_{\mathrm{Mn}}$ acceptors, for Fermi level $\left(E_{F}\right)$ positions close to the valence band. Thus, a comparable number of donors may be present at this equilibrium $E_{F}$, so, overall, the material acts as a compensated semiconductor. Other possible defects (e.g., $\mathrm{Te}_{\mathrm{Mn}}$ ) are higher in energy, so they do not significantly contribute to the charge balance. Defect formation energies obtained using the SCAN and PBE $+\mathrm{U}$ functionals for NC- and WZ-MnTe are also reported in Figure S5 within the Supplemental Material [30]. Overall, these theoretical predictions are consistent with the experimental observation that the hole concentration in WZ-MnTe is several orders of magnitude below that in NC-MnTe.

\section{CONCLUSIONS}

In the future, WZ-MnTe polymorph with a wide band gap and low doping can be applied as a channel layer in transistors used for transparent electronics [34]. The low doping levels are desirable for achieving low off-state current density and, hence, high on:off ratios, and the wide band gap implies transparency in the visible region of the optical spectrum. In addition to these two basic properties, the channel layer must also possess low densities of tail states near the band edges and trap states in the gap [35], as well as have suitable band offsets with the gate insulator material. Further calculations and experiments to determine these properties are warranted. Other potential future applications of WZ-MnTe include intrinsic layers for photodetectors because its low carrier density would lead to high photosensitivity and because its wide band gap would lead to low thermal noise for reliable room-temperature operation [36]. For both transistors and photodetectors based on WZ-MnTe, NC-MnTe can be used as a $p$-type contact without the need for extrinsic doping. This may be possible to implement by depositing NC-MnTe at $400^{\circ} \mathrm{C}$ (Fig. 1), since at this temperature the underlying WZMnTe deposited at $300^{\circ} \mathrm{C}$ would remain stable (Figure S1 within the Supplemental Material [30]).

In summary, we demonstrate the growth of thick metastable materials using a simple sputtering technique on amorphous glass substrates covered with thin seed layers. A metastable wurtzite polymorph of MnTe with a thickness of $500 \mathrm{~nm}$ is sputtered on a 5 -nm-thick ZnTe layer at $300^{\circ} \mathrm{C}$. The templated growth is theoretically explained by small differences in the bulk polymorph energies and large differences in the interface strain energies. Compared with the ground-state nickeline MnTe structure with a $1.3 \mathrm{eV}$ band gap and $10^{19} \mathrm{~cm}^{-3}$ hole density, the metastable wurtzite polymorph shows a wide $2.7 \mathrm{eV}$ band gap and low $10^{12} \mathrm{~cm}^{-3}$ hole concentration, with a reasonable mobility of $1.4 \mathrm{~cm}^{2} / \mathrm{Vs}$ for $p$-type nanocrystalline thin films. The lower doping level of WZ-MnTe relative to that of $\mathrm{NC}-\mathrm{MnTe}$ is explained by the higher formation energy of acceptor-type $V_{\mathrm{Mn}}$ defects in this metastable polymorph. In contrast to the ground-state $\mathrm{NC}$ structure, this WZ polymorph is an attractive candidate for wide-band-gap (opto-)electronic devices, such as thin-film transistors or photodetectors. Overall, this work demonstrates an example of how new properties can be achieved in known materials by the synthesis of their metastable polymorphs.

\section{ACKNOWLEDGMENTS}

This work was authored at the National Renewable Energy Laboratory, operated by the Alliance for Sustainable Energy, LLC, under Contract No. DE-AC3608GO28308 with the U.S. Department of Energy (DOE). Funding is provided by the Office of Science, Office of Basic Energy Sciences, as part of the Energy Frontier Research Center "Center for Next Generation of Materials Design: Incorporating Metastability." This work uses high-performance computing resources located at NREL and sponsored by DOE-EERE. Y.H. acknowledges support the from Science and Technology Commission of Shanghai Municipality (Grant No. 16JC1400603) and a grant from the National Natural Science Foundation of China (Grant No. 61471126). Y.H. also thanks the China Scholarship Council for offering a stipend to perform research at the National Renewable Energy Laboratory. The authors would like to thank Sage Bauers, Angela Fioretti, Kevin Talley, and Yun $\mathrm{Xu}$ for useful discussions and valuable suggestions. The views expressed in the article do not necessarily represent the views of the DOE or the U.S. Government.

[1] A. Goyal and V. Stevanovi, Metastable rocksalt $\mathrm{ZnO}$ is $p$ type dopable, Phys. Rev. Mater. 2, 1 (2018).

[2] A. Facchetti, T. J. Marks, and Wiley InterScience (Online service), Transparent ElectronicsâĂr: From Synthesis to Applications (Wiley, Hoboken, 2010).

[3] A. de J. de Meux, G. Pourtois, J. Genoe, and P. Heremans, Defects in Amorphous Semiconductors: The Case of Amorphous Indium Gallium Zinc Oxide, Phys. Rev. Appl. 9, 54039 (2018).

[4] T. Y. Seong, J. Han, H. Amano, and H. Morkoc, III-Nitride Based Light Emitting Diodes and Applications (Springer, Heidelberg, 2013).

[5] S. Sheng, G. Fang, C. Li, S. Xu, and X. Zhao, p-type transparent conducting oxides, Phys. Status Solidi (a) 1900, 1891 (2006).

[6] G. Hautier, A. Miglio, G. Ceder, G.-M. Rignanese, and $\mathrm{X}$. Gonze, Identification and design principles of low hole effective mass $p$-type transparent conducting oxides, Nat. Commun. 4, 2292 (2013). 
[7] J. B. Varley, A. Miglio, V. A. Ha, M. J. Van Setten, G. M. Rignanese, and G. Hautier, High-throughput design of nonoxide $p$-type transparent conducting materials: Data mining, search strategy, and identification of boron phosphide, Chem. Mater. 29, 2568 (2017).

[8] O. Y. Gorbenko, S. V. Samoilenkov, I. E. Graboy, and A. R. Kaul, Epitaxial stabilization of oxides in thin films, Chem. Mater. 33, 4026 (2002).

[9] E. S. Machlin and P. Chaudhari, Theory of "pseudomorphic stabilization" of metastable phases in thin film form, Synth. Prop. Metastable Phases 11, 11 (1980).

[10] D. G. Schlom, L. Q. Chen, X. Pan, A. Schmehl, and M. A. Zurbuchen, A thin film approach to engineering functionality into oxides, J. Am. Ceram. Soc. 91, 2429 (2008).

[11] D. G. Schlom, L. Q. Chen, C. B. Eom, K. M. Rabe, S. K. Streiffer, and J. M. Triscone, Strain tuning of ferroelectric thin films, Annu. Rev. Mater. Res. 37, 589 (2007).

[12] V. Rawat, D. Zakharov, E. A. Stach, and T. D. Sands, Pseudomorphic stabilization of rocksalt $\mathrm{GaN}$ in TiN/GaN multilayers and superlattices, Phys. Rev. B 80, 024114 (2009).

[13] V. Pankov, M. Evstigneev, and R. H. Prince, Role of substrate in the pseudomorphic stabilization of rocksalt-type AlN phase in AlN/TiN superlattices, Appl. Phys. Lett. 80, 4142 (2002).

[14] J. Küther, R. Seshadri, W. Knoll, and W. Tremel, Templated growth of calcite, vaterite and aragonite crystals onselfassembled monolayers of substituted alkylthiols on gold, J. Mater. Chem. 8, 641 (1998).

[15] B. R. Heywood and S. Mann, Template-Directed Nucleation and Growth of Inorganic Materials, Adv. Mater 6, 9 (1994).

[16] A. Z. Sebastian Siol, Yanbing Han, John Mangum, Philip Schulz, Aaron M. Holder, Talysa R. Klein, Maikel F. A. M. van Hest, and Brian Gorman, Stabilization of wide band-gap $p$-type wurtzite MnTe thin films on amorphous substrates, J. Mater. Chem. C 6, 6297 (2018).

[17] Y. Han, A. M. Holder, S. Siol, S. Lany, Q. Zhang, and A. Zakutayev, Zinc-Stabilized manganese telluride with wurtzite crystal structure, J. Phys. Chem. C 122, 18769 (2018).

[18] S. Siol, A. Holder, J. Steffes, L. T. Schelhas, K. H. Stone, L. Garten, J. D. Perkins, P. A. Parilla, M. F. Toney, and B. D. Huey, Negative-pressure polymorphs made by heterostructural alloying, Sci. Adv. 4, 1442 (2018).

[19] K. R. Talley, S. R. Bauers, C. L. Melamed, M. C. Papac, K. N. Heinselman, I. Khan, D. M. Roberts, V. Jacobson, A. Mis, and G. L. Brennecka, Combigor: Data analysis package for combinatorial materials science, ACS Comb. Sci. 21, 537 (2019).

[20] A. Zakutayev, N. Wunder, M. Schwarting, J. D. Perkins, R. White, K. Munch, W. Tumas, and C. Phillips, An open experimental database for exploring inorganic materials, Sci. Data 5, 180053 (2018).
[21] S. Siol, T. P. Dhakal, G. S. Gudavalli, P. P. Rajbhandari, C. Dehart, L. L. Baranowski, and A. Zakutayev, Combinatorial reactive sputtering of $\operatorname{In}_{2} \mathrm{~S}_{3}$ as an alternative contact layer for thin film solar cells, ACS Appl. Mater. Interfaces 8, 14004 (2016).

[22] G. Kresse and J. Furthmüller, Self-interaction correction to density functional approximation for many electron systems, Phys. Rev. B 54, 11169 (1996).

[23] G. Kresse and D. Joubert, From ultrasoft pseudopotentials to the projector augmented-wave method, Phys. Rev. B 59, 1758 (1999).

[24] P. E. Blöchl, Projector augmented-wave method, Phys. Rev. B 50, 17953 (1994).

[25] J. Sun, A. Ruzsinszky, and J. P. Perdew, Strongly Constrained and Appropriately Normed Semilocal Density Functiona, Phys. Rev. Lett. 115, 36402 (2015).

[26] J. P. Perdew, K. Burke, and M. Ernzerhof, Generalized Gradient Approximation Made Simple, Phys. Rev. Lett. 77, 3865 (1996).

[27] S. L. Dudarev, G. A. Botton, S. Y. Savrasov, C. J. Humphreys, and A. P. Sutton, Electron-energy-loss spectra and the structural stability of nickel oxide: An LSDA+ U study, Phys. Rev. B 57, 1505 (1998).

[28] V. Stevanović, S. Lany, X. Zhang, and A. Zunger, Correcting density functional theory for accurate predictions of compound enthalpies of formation: Fitted elemental-phase reference energies, Phys. Rev. B 85, 115104 (2012).

[29] S. Lany and A. Zunger, Assessment of correction methods for the band-gap problem and for finite-size effects in supercell defect calculations: Case studies for $\mathrm{ZnO}$ and GaAs, Phys. Rev. B 78, 235104 (2008).

[30] See the Supplemental Material at http://link.aps.org/supp lemental/10.1103/PhysRevApplied.13.014012 for more structure, microscopy, templated growth, optics, and defect information.

[31] A. Zakutayev, C. M. Caskey, A. N. Fioretti, D. S. Ginley, J. Vidal, V. Stevanovic, E. Tea, and S. Lany, Defect tolerant semiconductors for solar energy conversion, J. Phys. Chem. Lett. 5, 1117 (2014).

[32] A. Rajan, R. T. Moug, and K. A. Prior, Growth and stability of zinc blende $\mathrm{MgS}$ on GaAs, GaP, and InP substrates, Appl. Phys. Lett. 102, 32102 (2013).

[33] S. Havelia, S. Wang, K. R. Balasubramaniam, A. M. Schultz, G. S. Rohrer, and P. A. Salvador, Combinatorial substrate epitaxy: A new approach to growth of complex metastable compounds, CrystEngComm 15, 5434 (2013).

[34] J. F. Wager, D. A. Keszler, and R. E. Presley, Transparent Electronics (Springer, New York, 2008).

[35] H. Kumomi, T. Kamiya, and H. Hosono, Advances in oxide thin-film transistors in recent decade and their future, ECS Trans. 67, 3 (2015).

[36] E. Monroy, F. Omnès, and F. Calle, Wide-bandgap semiconductor ultraviolet photodetectors, Semicond. Sci. Technol. 18, R33 (2003). 